\title{
Partial left ventriculectomy improves left ventricular end systolic elastance in patients with idiopathic dilated cardiomyopathy
}

\author{
Z Popović, M Mirić, S Gradinac, A N Nešković, M Bojić, A D Popović
}

\begin{abstract}
Objective-To assess the effect of partial left ventriculectomy (PLV) on estimate of left ventricular end systolic elastance (Ees), arterial elastance, and ventriculoarterial coupling.

Patients-11 patients with idiopathic dilated cardiomyopathy before and two weeks after PLV, and 11 controls.

Interventions-Single plane left ventricular angiography with simultaneous measurements of femoral artery pressure was performed during right heart pacing before and after load reduction. Results-PLV increased mean (SD) Ees from $0.52 \quad(0.27)$ to $1.47 \quad(0.62) \mathrm{mm} \mathrm{Hg} / \mathrm{ml}$ $(\mathrm{p}=0.0004)$. The increase in Ees remained significant after correction for the change in left ventricular mass $(p=0.004)$ and end diastolic volume $(p=0.048)$. As PLV had no effect on arterial elastance, ventriculoarterial coupling improved from $3.25(2.17)$ to $1.01(0.93)(p=0.017)$, thereby maximising left ventricular stroke work.

Conclusion-It appears that PLV improves both Ees and ventriculoarterial coupling, thus increasing left ventricular work efficiency.

(Heart 2000;83:316-319)
\end{abstract}

Keywords: dilated cardiomyopathy, elastance, partial left ventriculectomy

Previous studies have shown that partial left ventriculectomy (PLV), a surgical procedure originally described by Batista, ${ }^{1}$ leads to a significant reduction in left ventricular volume while simultaneously increasing ejection fraction. ${ }^{2-4}$ Mathematical modelling also predicts that volume reduction by PLV increases left ventricular end systolic elastance ${ }^{5}$ - that is, the steepness of end systolic pressure-volume relations. ${ }^{6}$ However, clinical data on the effects of PLV on this load independent measure of left ventricular contractility are controversial. ${ }^{7}$

We report the effects of PLV on left ventricular end systolic elastance in patients with severely symptomatic idiopathic dilated cardiomyopathy.

\section{Methods}

PATIENTS

We assessed 11 male patients that represented a subgroup of 19 previously reported ${ }^{2}$ patients who underwent successful PLV with technically satisfactory data for estimating pre- and postoperative left ventricular end systolic elastance. In eight patients end systolic elastance data could not be calculated because of technical reasons and they were not included in this analysis. (In seven patients only one adequate preoperative ventricular angiogram was obtained because of the following: four patients had extrasystoles, in two patients there was inadequate opacification of the left ventricle, and one patient had an inadequate pressure drop after glyceryl trinitrate. In one patient tachycardia occurred before the second postoperative angiogram.) All 11 patients had chronic heart failure caused by idiopathic dilated cardiomyopathy, severe left ventricular dilation (echocardiographic end diastolic di- mension $>70 \mathrm{~mm}$ ) with normal coronary arteries, normal valve morphology, and no history of alcohol abuse. Preoperative patient characteristics were similar between these patients and eight patients in whom end systolic elastance estimates could not be calculated. All patients were treated with digoxin, captopril, amiodarone, and frusemide (furosemide), both pre- and postoperatively. No patient was on inotropic support during pre- or postoperative catheterisation studies. The investigational status of PLV was explained to all patients, who signed informed consent forms both for the catheterisation studies and for the operation.

A control group comprised 11 patients with atypical chest pain and normal coronary arteries in whom data for calculation of end systolic elastance estimates were collected. None of these patients had a history of hypertension, while in all of them echocardiography showed normal left ventricular size (end diastolic diameter $<56 \mathrm{~mm}$ ) with no signs of valvar heart disease or left ventricular hypertrophy (wall thickness $<11 \mathrm{~mm}$ ).

\section{SURGICAL AND ANGIOGRAPHIC PROCEDURES}

The surgical technique used has been previously described. ${ }^{9}$ All operations were performed using cardiopulmonary bypass. In 2/11 patients beating heart technique was used, while in $9 / 11$ patients surgery was performed during cardioplegic arrest. Mitral valve repairs were performed using a previously described modification. ${ }^{9}$ The procedure involved a diamond shaped resection of the left ventricular posterolateral wall confined by two papillary muscles. The resected myocardial specimen was $9.1(2.5) \mathrm{cm}$ long, 4.5 (1.3) $\mathrm{cm}$ wide, and 
Table 1 Clinical and haemodynamic data

\begin{tabular}{|c|c|c|c|c|c|c|c|c|c|c|c|c|}
\hline \multirow[b]{2}{*}{ Patient } & \multirow{2}{*}{$\begin{array}{l}\text { Age } \\
\text { (years) }\end{array}$} & \multirow{2}{*}{$\begin{array}{l}\text { NYHA } \\
\text { class }\end{array}$} & \multirow[b]{2}{*}{ Rhythm } & \multirow[b]{2}{*}{ Valve procedures } & \multicolumn{2}{|c|}{ Ees $(m m ~ H g / m l)$} & \multicolumn{2}{|c|}{ Eps $(m m H g / m l)$} & \multicolumn{2}{|c|}{$\mathrm{Ea}(\mathrm{mm} H \mathrm{Hg} / \mathrm{ml})$} & \multicolumn{2}{|c|}{ Ea/Ees } \\
\hline & & & & & Pre & Post & Pre & Post & Pre & Post & Pre & Post \\
\hline 1 & 59 & IV & $\mathrm{AF}$ & CS & 0.43 & 0.87 & 0.484 & 1.052 & 1.02 & 1.04 & 2.36 & 1.20 \\
\hline 2 & 66 & IV & $\mathrm{AF}$ & $\mathrm{MR}, \mathrm{CS}$ & 0.38 & 0.68 & 0.225 & 1.021 & 0.91 & 1.12 & 2.43 & 1.64 \\
\hline 3 & 46 & IV & NSR & $\mathrm{MR}, \mathrm{CS}$ & 0.24 & 0.56 & 0.21 & 0.421 & 1.70 & 0.76 & 7.00 & 1.35 \\
\hline 4 & 38 & IV & NSR & $\mathrm{CS}$ & 0.70 & 2.05 & 0.701 & 2.432 & 1.25 & 1.38 & 1.78 & 0.67 \\
\hline 5 & 47 & IV & NSR & TA, MVR & 0.20 & 1.88 & 0.807 & 2.939 & 0.58 & 0.73 & 2.88 & 0.39 \\
\hline 6 & 56 & IV & NSR & $\mathrm{TA}, \mathrm{MR}, \mathrm{CS}$ & 0.72 & 2.43 & 1.26 & 3.138 & 2.18 & 0.78 & 3.02 & 0.32 \\
\hline 7 & 50 & III & NSR & $\mathrm{MR}, \mathrm{CS}$ & 0.48 & 1.79 & 0.536 & 1.879 & 1.40 & 0.91 & 2.91 & 0.51 \\
\hline 8 & 62 & IV & $\mathrm{AF}$ & TA, MR, CS & 0.40 & 0.89 & 0.41 & 0.721 & 0.93 & 1.62 & 2.34 & 1.82 \\
\hline 9 & 56 & IV & $\mathrm{AF}$ & $\mathrm{TA}, \mathrm{MR}, \mathrm{CS}$ & 1.03 & 1.50 & 1.141 & 1.782 & 1.38 & 1.85 & 1.34 & 1.24 \\
\hline 10 & 58 & III & $\mathrm{AF}$ & TA, MVR & 0.84 & 1.86 & 0.404 & 1.888 & 1.46 & 1.24 & 1.72 & 0.67 \\
\hline 11 & 41 & IV & NSR & TA, MVR & 0.24 & 1.69 & 0.195 & 1.771 & 1.94 & 2.21 & 7.96 & 1.31 \\
\hline Mean & 53 & & & & $0.52 \dagger$ & $1.47 \S$ & $0.58 \dagger$ & $1.73 \star \delta$ & 1.34 & 1.24 & $3.25+$ & $1.01^{\star} \ddagger$ \\
\hline $\mathrm{SD}$ & 9 & & & & 0.27 & 0.62 & $0.36^{\circ}$ & 0.88 & 0.47 & 0.49 & $2.17^{\circ}$ & 0.52 \\
\hline \multicolumn{13}{|l|}{ Controls } \\
\hline Mean (SD) & \multicolumn{4}{|l|}{$52(7)$} & \multicolumn{2}{|c|}{$3.27(2.29)$} & \multicolumn{2}{|c|}{$4.04(2.65)$} & \multicolumn{2}{|c|}{$1.16(0.37)$} & \multicolumn{2}{|c|}{$0.52(0.32)$} \\
\hline
\end{tabular}

${ }^{\star} \mathrm{p} \leqslant 0.05, \dagger \mathrm{p} \leqslant 0.01$ versus control; $\ddagger \mathrm{p} \leqslant 0.05, \$ \mathrm{p} \leqslant 0.001$ versus pre. AF, atrial fibrillation; CS, coaptation stitch across the mitral valve; Ea, arterial elastance (mm $\mathrm{Hg} / \mathrm{ml}$ ); Ea/Ees, ventriculoarterial coupling; Ees, first end systolic elastance estimate; Eps, second end systolic elastance estimate; MR, mitral valve repair; MVR, mitral valve replacement; NSR, normal sinus rhythm; NYHA, New York Heart Association; TA, tricuspid annnuloplasty.

$1.8(0.7) \mathrm{cm}$ thick. Additional valve procedures are presented in table 1 .

All patients underwent preoperative (15 (10) days before surgery) and postoperative (13 (3) days after surgery) catheterisation studies. The control group was evaluated using the same protocol as for the study patients. Cardiovascular medications were not withheld on the day of the procedure. After recording baseline
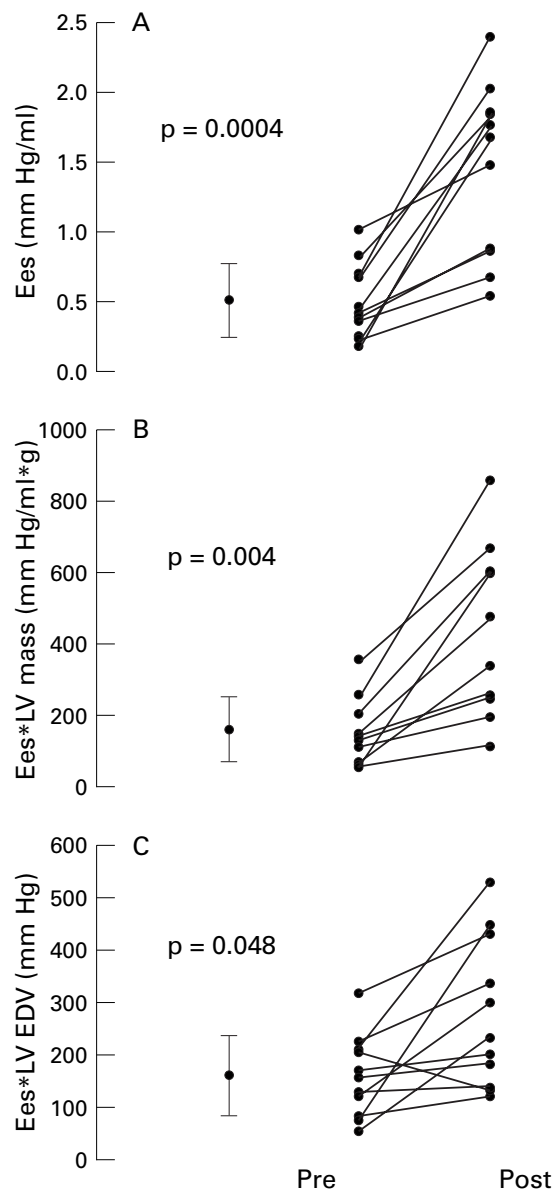

Figure 1 Effect of PLV on Ees, an estimate of left ventricular end systolic elastance. There was a substantial improvement of Ees $(p=0.0004)(A)$. Improvement of Ees by PLV persisted after multiplying Ees with left ventricular mass (Ees,LV mass) $(p=0.004)(B)$, and after multiplying Ees with left ventricular end diastolic volume (Ees.LV EDV) $(p=0.048)(C)$. intracardiac pressures and cardiac output, and performing coronary angiography, patients entered a modified version of previously reported protocols. ${ }^{10}{ }^{11} \mathrm{~A}$ bipolar pacing catheter was placed into the right atrium (or into the right ventricle in patients with atrial fibrillation). Pacing was used to avoid differences caused by cycle length during ventricular angiographies. A $7 \mathrm{~F}$ pigtail catheter was inserted into the left ventricle through an $8 \mathrm{~F}$ femoral sheath. Single plane left ventricular angiography was performed in $30^{\circ}$ right anterior oblique view using $35 \mathrm{~mm}$ film at a rate of 50 frames/s, after the injection of $40-50 \mathrm{ml}$ of non-ionic contrast medium. Femoral artery pressure was recorded during left ventricular angiography using a side arm of a femoral sheath at a paper speed of $25 \mathrm{~mm} / \mathrm{s}$ on a Mingograf 7 strip chart recorder (Siemens, Erlangen, Germany). After allowing for adequate recovery time to achieve a return of stable baseline haemodynamics, an intravenous glyceryl trinitrate bolus was administered into a femoral vein to decrease systolic arterial pressure by at least $20 \mathrm{~mm} \mathrm{Hg}$ or to a level of $80 \mathrm{~mm} \mathrm{Hg} .{ }^{12}$ At that moment, a second left ventricular angiography was performed.

ANGIOGRAPHIC ANALYSIS PROCEDURES

For each ventricular angiogram, left ventricular volumes and femoral artery pressures of the first two heart cycles, which provided adequate left ventricular opacification and were not postextrasystolic, were averaged and used for the analysis. Left ventricular volumes were calculated by the area-length method using an appropriate regression equation. ${ }^{13}$ We previously reported the accuracy of single plane ventricular angiography for the assessment of left ventricular volumes in this patient population. ${ }^{2}$ End diastolic and end systolic frames were defined as the frames with the largest and smallest ventricular silhouette, respectively.

Correlations between intraobserver variability of measurements of left ventricular end diastolic and end systolic volumes, and for the end systolic volume decrease after glyceryl trinitrate bolus in our patient group, were $r=0.97,0.98$, and 0.76, respectively. The 

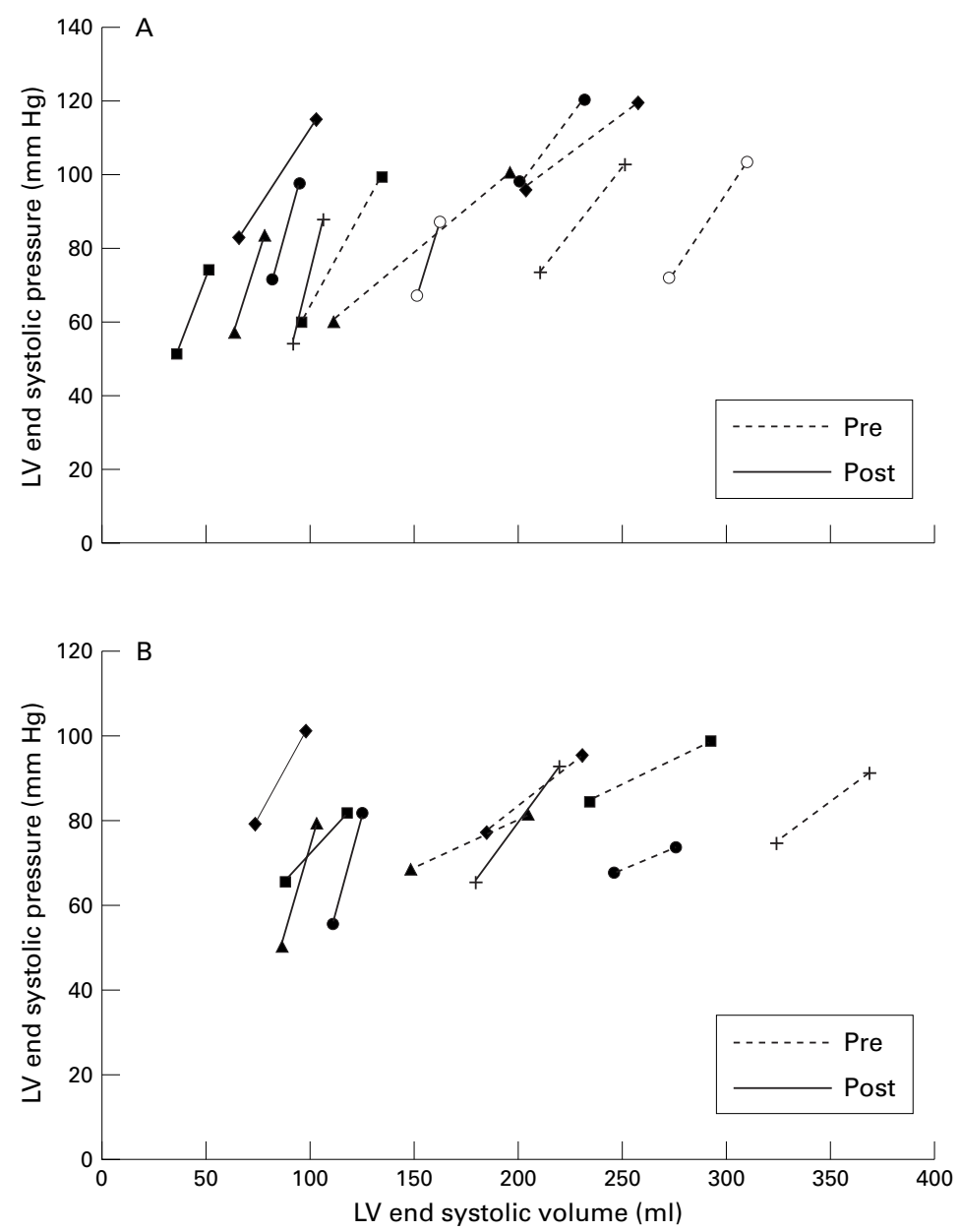

Figure 2 Individual left ventricular (LV) end systolic pressure-volume relations in $(A)$ patients with no or mild mitral regurgitation $(n=6)$, and $(B)$ patients with severe mitral regurgitation $(n=5)$. End systolic elastance was higher in patients with no or mild mitral regurgitation both pre- and postoperatively $(p=0.02$ by analysis of variance (ANOVA)), while its increase was similar in both groups ( $p>0.05$ by ANOVA). Dashed lines denote preoperative values, whereas solid lines denote postoperative values. The markers are the same for individual patients pre- and postoperatively.

correlation between interobserver variability of measurements of the same variables were $r=0.95,0.96$, and 0.72 , respectively. The mean (SD) of absolute differences of intraobserver measurements of left ventricular end diastolic and end systolic volumes and for the end systolic volume decrease after the glyceryl trinitrate bolus were $19.0(14.1) \mathrm{ml}, 10.5$ (11.0) $\mathrm{ml}$, and $11.8(10.1) \mathrm{ml}$, respectively; the mean of absolute differences of interobserver measurements of the same variables were 23.6 (20.7) $\mathrm{ml}, 17.3(13.6) \mathrm{ml}$, and 11.1 (7.4) $\mathrm{ml}$, respectively.

End systolic pressure was defined as the dicrotic notch of the femoral artery pressure tracing recorded during left ventricular angiography. ${ }^{2}$ Left ventricular end diastolic wall thickness was measured as the distance between epicardial and endocardial surfaces of the anterior free wall in its middle third, and the result was used for determination of ventricular mass. ${ }^{14}$

Left ventricular end systolic elastance was estimated by two methods. First, the matched points of end systolic pressure-volume relation were subjected to a linear regression. The regression slope was considered to be the first end systolic elastance estimate (Ees), while its intercept with the abscissa was considered to be the theoretical volume at zero pressure. ${ }^{6} 1011$ The end systolic pressure was then replaced with peak systolic pressure and the method repeated, with a resulting regression slope considered as a second end systolic elastance estimate (Eps). Previous studies have shown a good correlation between these two estimates and more accurate measures of left ventricular end systolic elastance. ${ }^{15}$ To eliminate the impact of change of left ventricular size on end systolic elastance, we calculated corrected Ees by multiplying it with either left ventricular mass or end diastolic volume. ${ }^{16}{ }^{17}$ Arterial elastance was calculated as the ratio of end systolic pressure to stroke volume, and ventriculoarterial coupling was calculated by dividing arterial elastance with Ees. ${ }^{18}$

STATISTICS

All data are presented as mean (SD). Paired $t$ tests were used to compare patients' data before and after surgery and unpaired $t$ tests were used for comparisons between controls and patients, using Bonferroni's correction for multiple comparisons. Simple linear regression was used to assess correlation between variables. The impact of correction of mitral regurgitation on Ees was assessed by repeated measures analysis of variance. A value of $\mathrm{p} \leqslant 0.05$ was considered significant.

\section{Results}

Following PLV, left ventricular mass index significantly decreased from 163 (24) to 141 (27) $\mathrm{g} / \mathrm{m}^{2},(\mathrm{p}=0.001)$; this corresponded to a relative decrease of left ventricular mass of 14.4 (7.0)\%. This was associated with a decrease of end diastolic volume from 172 (45) to 101 (32) $\mathrm{ml} / \mathrm{m}^{2} \quad(\mathrm{p}<0.0001)$ and of end systolic volume from 129 (36) to 59 (24) $\mathrm{ml} / \mathrm{m}^{2}$ $(p<0.0001)$. We also found a significant increase in ejection fraction from 25 (7) to 42 $(10) \%(p=0.0006)$. An improvement of Ees after PLV was detected in all patients, from $\begin{array}{lll}0.52(0.27) & \text { to } 1.47 \quad(0.62) \mathrm{mm} \mathrm{Hg} / \mathrm{ml}\end{array}$ $(\mathrm{p}=0.0004)$ (fig 1). Similar improvements were obtained for Eps, from $0.58(0.36)$ to 1.73 (0.88) $\mathrm{mm} \mathrm{Hg} / \mathrm{ml}(\mathrm{p}=0.002)$ (table 1). There was a good overall correlation between Ees and Eps $(r=0.95, p<0.0001)$. In addition, there was a significant linear correlation between the logarithm of Ees and the ejection fraction $(r=0.77, \mathrm{p}<0.0001)$. The increase of Ees remained significant after correction for the change of left ventricular mass, from 163 (91) to $420(238) \mathrm{mm} \mathrm{Hg} / \mathrm{ml}_{\star} \mathrm{g}(\mathrm{p}=0.004)$, as well as after correction for the change of end diastolic volume (from 165 (77) to 281 (141) $\mathrm{mm} \mathrm{Hg}(p=0.048)$. There was no change in theoretical volume at zero pressure (11.3 (102.5) v 37.6 (49.5) ml, $\mathrm{p} \mathrm{>} \mathrm{0.05),} \mathrm{and}$ in arterial elastance (1.36 (0.46) $v 1.18$ $(0.50) \mathrm{mm} \mathrm{Hg} / \mathrm{ml}, \mathrm{p}>0.05)$ after surgery. Finally, an improvement in ventriculoarterial coupling (from 3.25 (2.17) to 1.01 (0.93), $\mathrm{p}=0.017$ ) occurred after surgery.

To clarify the impact of surgical correction of mitral regurgitation on Ees change after PLV, 
we divided patients into two groups: a group with no or mild preoperative mitral regurgitation (grade $<2$ by ventricular angiography, $\mathrm{n}=6$ ), and a group with severe mitral regurgitation (grade $>2, n=5$ ) (fig 2). Compared to patients with no or mild mitral regurgitation, patients with severe mitral regurgitation had lower values of Ees both before $(0.29(0.22) v$ $0.70(0.09) \mathrm{mm} \mathrm{Hg} / \mathrm{ml})$ and after PLV (1.14 $(0.53) v 1.74(0.60) \mathrm{mm} \mathrm{Hg} / \mathrm{ml})(\mathrm{p}=0.02)$. However, the amount of postoperative increase in Ees in both groups was similar $(p>0.05)$.

\section{Discussion}

Published data on the effects of PLV on left ventricular end systolic elastance are controversial. While Bellotti and colleagues ${ }^{7}$ found a postoperative increase of end systolic elastance (using $\mathrm{M}$ mode echocardiography coupled with simultaneous measurement of left ventricular pressures), Gorcsan and colleagues ${ }^{8}$ reported variable intraoperative effects of PLV on end systolic elastance. The results of Gorcsan and colleagues ${ }^{8}$ may have been influenced by immediate postoperative left ventricular depression, as well as by a small number of patients with both pre- and postoperative data.

In contrast, we have found a significant improvement in end systolic elastance. End systolic elastance reflects the ability of the left ventricle to resist distending forces generated by an increase of systolic pressure. ${ }^{6}$ This measure of left ventricular contractility gains importance in a setting of decreased end diastolic volume after PLV. Two recent papers ${ }^{5}{ }^{19}$ predicted an increase of end systolic elastance following ventricular volume reduction by using different methods of mathematical modelling. Thus, a decrease of end systolic elastance postoperatively would imply deleterious effects of PLV procedure on left ventricular function. Therefore, a similar or decreased postoperative end systolic elastance would seriously challenge proposed beneficial effects of PLV. In our study, all patients had a significant increase of two Ees. Interestingly, the magnitude of this increase was greater than anticipated and it was still significant after correction for changes of left ventricular mass and end diastolic volume. ${ }^{16}$ This may suggest that postoperative change of Ees is not related to the change of left ventricular size only, but to the change of its shape as well. ${ }^{2} 7$

Our data indicate that PLV improves Ees. In addition, this increase improves postoperative ventriculoarterial coupling, suggesting better left ventricular pump efficiency, ${ }^{18}$ which may be responsible for the beneficial haemodynamic effects of PLV. ${ }^{5}$

1 Batista RJ, Verde J, Nery P, et al. Partial left ventriculectomy to treat end-stage heart disease. Ann Thorac Surg 1997;64:634-8

2 Popović Z, Mirić M, Gradinac S, et al. Effects of partial left ventriculectomy on left ventricular performance in patients with nonischemic dilated cardiomyopathy. $f$ Am Coll Cardiol 1998;32:1801-8.

3 Bocchi EA, Belotti G, de Moraes AV, et al. Clinical outcome after left ventricular surgical remodeling in patients with idiopathic dilated cardiomyopathy referred for heart transplantation: short term results. Circulation 1997; 96(suppl II):II-65-72.

4 McCarthy PM, Starling RC, Wong J, et al. Early results with partial left ventriculectomy. F Thorac Cardiovasc Surg 1997;

5 Dickstein ML, Spotnitz HM, Rose EA, et al. Heart reduction surgery: an analysis of the impact on cardiac function. F Thorac Cardiovasc Surg 1997;114:1032-40

6 Kass DA, Maughan WL. From Emax to pressure-volume relations: a broader view. Circulation 1988;77:1203-12.

7 Bellotti G, Moraes A, Bocchi E, et al. Efeitos de ventriculectomia parcial nas propriedadeas mecanicas, forma e geometria de ventriculo esquerdo em portedores de cardi

8 Gorcsan J III, Feldman AM, Kormos RL, et al. Heterogenous immediate effects of partial left ventriculectomy on enous immediate effects of partial left ventriculect

9 Gradinac S, Mirić M, Popović Z, et al. Partial left ventriculectomy in patients with idiopathic dilated cardiomyopathy: early results and six-month follow-up. Ann Thorac Surg 1998;66:1963-8.

10 Grossman W, Braunwald E, Mann T, et al. Contractile state of the left ventricle in man as evaluated from the end-systolic pressure-volume relation. Circulation 1977;56: 845-52.

11 Mehmel HC, Stockins B, Ruffmann K, et al. The linearity of the end-systolic pressure-volume relationship in man and its sensitivity for assessment of left ventricular function. Circulation 1981;63:1216-22.

12 Heinrich $\mathrm{H}$. Systolic pressure dimension relationships and diastolic dimensions for monitoring of left ventricular function. In: Erbel R, Khandheria BK, Brennecke R, eds. Transesophageal echocardiography. Berlin: Springer Verlag 1989:274-81.

13 Wynne J, Green LH, Mann T, et al. Estimation of left ventricular volumes in man from biplane cineangiograms filmed in oblique projections. Am f Cardiol 1978;41:726-

14 Rackley CE, Dodge HT, Coble YD, et al. A method for determining left ventricular mass in man. Circulation 1964; 29:666-71.

15 Starling MR, Walsh RA, Dell'Italia LJ, et al. The relationship of various measures of end-systole to left ventricular maximum time-varying elastance in man. Circulatricular maximum tim

16 Mirsky I, Corin WJ, Murakami T, et al. Correction for preload in an assessment of myocardial contractility in aortic and mitral valve disease: an application of the concept of systolic myocardial stiffness. Circulation 1988;78:68-80.

17 Berko B, Gaasch WH, Tanigawa N, et al. Disparity between ejection and end-systolic indexes of left ventricular contractility in mitral regurgitation. Circulation 1987;75: 1310-19.

18 Asanoi H, Sasayama S, Kameyama T. Ventriculoarterial coupling in normal and failing hearts in humans. Circ Res 989;65:483-93.

19 Ratcliffe MB, Hong J, Salahieh A, et al. The effect of ventricular volume reduction surgery in the dilated, poorly contractile left ventricle: a simple finite element analysis. $\mathcal{F}$ Thorac Cardiovasc Surg 1998;116:566-77.

20 Kass DA. Ventriculectomy: a direct application of Laplace's law. Arq Bras Cardiol 1996;67:401-2. 